\title{
Total Synthesis of Hematoporphyrin and Protoporphyrin; a Conceptually New Approach
}

\author{
Pierre Martin*, Markus Müller, Dietmar Flubacher, Andreas Boudier, and Dirk Spielvogel \\ Winners of the Sandmeyer Award sponsored by KPMG 2012 \\ In memoriam Prof. Dr. H. Prinzbach
}

\begin{abstract}
The total synthesis of protoporphyrin IX and its disodium salt using a new alternative method to the classical MacDonald condensation is reported. The key step is the reaction of the new unsymmetrical diiodo dipyrrylmethane $\mathbf{1}$ with the known dipyrrylmethane $\mathbf{2}$. Coupling of the two fragments leads directly to porphyrin $\mathbf{3}$ without the need of an oxidizing agent. The new methodology is well suited for the synthesis of protoporphyrin IX derivatives on a multi-100 g scale in good quality without the need for chromatography. Furthermore, these preparations are completely free of any contaminant of animal origin, which represents a real improvement in the manufacturing of protoporphyrin IX derivatives.
\end{abstract}

Keywords: Development \& scale-up · Novel porphyrin cyclization method · Process research · Protoporphyrin IX · Total synthesis

\section{Introduction}

Protoporphyrin IX (Fig. 1) is most commonly prepared by semisynthesis starting from hemin, which in turn is isolated from ox or pork blood. ${ }^{[1,2]}$ For medical applications, e.g. in cell culture media, this poses the problem of impurities of animal origin that may be present, in particular of agents in connection with transmissible spongiform encephalopathy. In order to meet the guidelines of the regulatory agencies, ${ }^{[3]}$ it was necessary to provide a completely synthetic preparation process for the preparation of protoporphyrin IX that uses only products of synthetic origin.

Here, we describe the total synthesis of protoporphyrin IX and its disodium salt using a novel variant of the MacDonald condensation method. The key step is the reaction of the new unsymmetrical diiodo-
${ }^{\star}$ Correspondence: Dr. P. Martin Solvias AG

Römerpark 2, CH-4303 Kaiseraugst E-mail: pierre.martin@solvias.com dipyrrylmethane $\mathbf{1}$ with the known dipyrrylmethane 2 (Scheme 1). [4] The two fragments are coupled without the presence of a metal leading directly to porphyrin 3 without the need of an oxidizing agent. The new methodology is well suited for the synthesis of protoporphyrin IX on a multi-100 g scale in good quality without the need for chromatography.

\section{Synthetic Concept}

The classical MacDonald condensation where a dipyrrylmethane unit is reacted with a dipyrrylmethane dialdehyde has been widely applied for the preparation of a variety of unsymmetrically substituted porphyrins. ${ }^{[5]}$ The primary product is a dihydroporphin which must then be oxidized to obtain the corresponding porphyrin using either air ${ }^{[5]}$ or a quinone such as DDQ. ${ }^{6]}$ In many cases, the oxidation step is rather problematic since the dihydroporphin

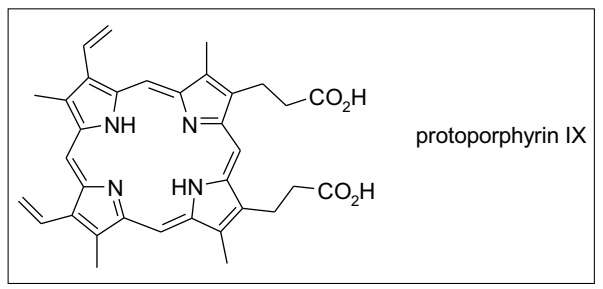

Fig. 1. is quite unstable and not easy to detect. Furthermore, the reduced quinone is difficult to remove from the reaction mixture requiring a chromatographic separation. For these reasons, scale up problems are unavoidable and, indeed, literature preparations are usually described in the $\mathrm{mg}$ range.

We reasoned that in order to avoid the formation of the dihydroporphin, one of the two dipyrrylmethanes should either be in a higher oxidation state or carry a nucleofuge which can be eliminated to form the required additional $\mathrm{C}=\mathrm{C}$ double bond. We decided to prepare the diiodo derivative 1 and to react it under MacDonald conditions with dipyrrylmethane dialdehyde 2 (Scheme 1). To our delight and surprise, we directly obtained the desired porphyrin $\mathbf{3}$ in good yields. We postulate that the primary condensation product is intermediate A which spontaneously eliminates water as well as one mol of HI to give the iodinated porphyrin $\mathbf{B}$ which then is reduced by the HI formed in the first step to give $\mathbf{3}$ and $\mathrm{I}_{2}{ }^{\left[{ }^{[7]} \mathrm{We}\right.}$ consider the elimination of $2 \mathrm{~mol}$ of HI improbable since a rather thermodynamically unfavourable dehydroporphyrin would be formed.

\section{Synthetic Details[8]}

The synthesis of the new diiodo dipyrrylmethane 1 was carried out as depicted 
in Scheme 2. The partially optimized synthesis which delivered the desired intermediate in an acceptable over-all yield, starts with the silylated propyne $\mathbf{4}$ which is reacted with acetyl chloride in presence of $\mathrm{AlCl}_{3}$ to give 3-pentyne-2-one (5). The phosphine-catalyzed reaction of $\mathbf{5}$ with benzyl isocyanoacetate gave the first pyrrole unit $\mathbf{6}$ which was obtained in pure form after chromatography in moderate yield. The second pyrrole moiety 9 was prepared starting from the commercially available pyrrole ethyl ester 7 which was first transformed to the corresponding benzyl ester 8 with $\mathrm{Na} / \mathrm{BnOH}$ at $190{ }^{\circ} \mathrm{C}$ and then acetoxylated in presence of sulfuryl chloride to give 9 in moderate yield. The condensation of the two pyrrolo units 6 and 9 catalyzed by $\mathrm{HBF}_{4}$ gave the desired dipyrrylmethane $\mathbf{1 0}$ in an acceptable yield of $68 \%$. After removal of the benzyl groups via hydrogenolysis with $\mathrm{Pd} / \mathrm{C}$ to give the diacid 11, the novel diiodo dipyrrylmethane 1 was obtained in excellent yield and high purity as light red crystals.

The synthesis of the second dipyrrylmethane moiety 2 was carried out as depicted in Scheme 3. Commercially available 12 was brominated selectively and then condensed with the loss of a $\mathrm{C}_{1}$ unit to give dipyrrylmethane $\mathbf{1 3}$ in good yield. Dialdehyde $\mathbf{2}$ was then obtained in excellent yield via debenzylation with $\mathrm{Pd} / \mathrm{C}$ to give 14, followed by reduction with trimethyl orthoformate in presence of trifluoroacetic acid.

With the two dipyrrylmethane moieties $\mathbf{1}$ and $\mathbf{2}$ in hand, the condensation reaction was carried out in a mixture of acetic acid, acetic anhydride and trifluoromethyl sulfonic acid (Scheme 1). The desired porphyrin 3 was indeed obtained in excellent $76 \%$ yield. The target protoporphyrin IX (16) and its disodium salt $\mathbf{1 7}$ were obtained as shown in Scheme 4 via reduction of the two acetyl groups with $\mathrm{NaBH}_{4}$ to give the hematoporphyrin ester $\mathbf{1 5}$, followed by elimination of the intermediately formed dibenzoates in good to excellent yields. While 16 was obtained as violet-black crystals of high purity, the disodium salt was isolated as an amorphous powder.

As can be seen from the detailed experimental part, the synthesis of $\mathbf{1 7}$ was first carried out on a $6 \mathrm{~g}$ scale. While most intermediates could be isolated via distillation or crystallization some compounds had to be purified by column chromatography. In an intensive process development, all of these chromatographic steps could be circumvented by relatively small variations of the experimental conditions. The improved procedure was then applied to the preparation of protoporphyrin IX derivatives on a $100 \mathrm{~g}$ scale with an overall yield in the range of 40\% starting from $\mathbf{1}$ and 2 .

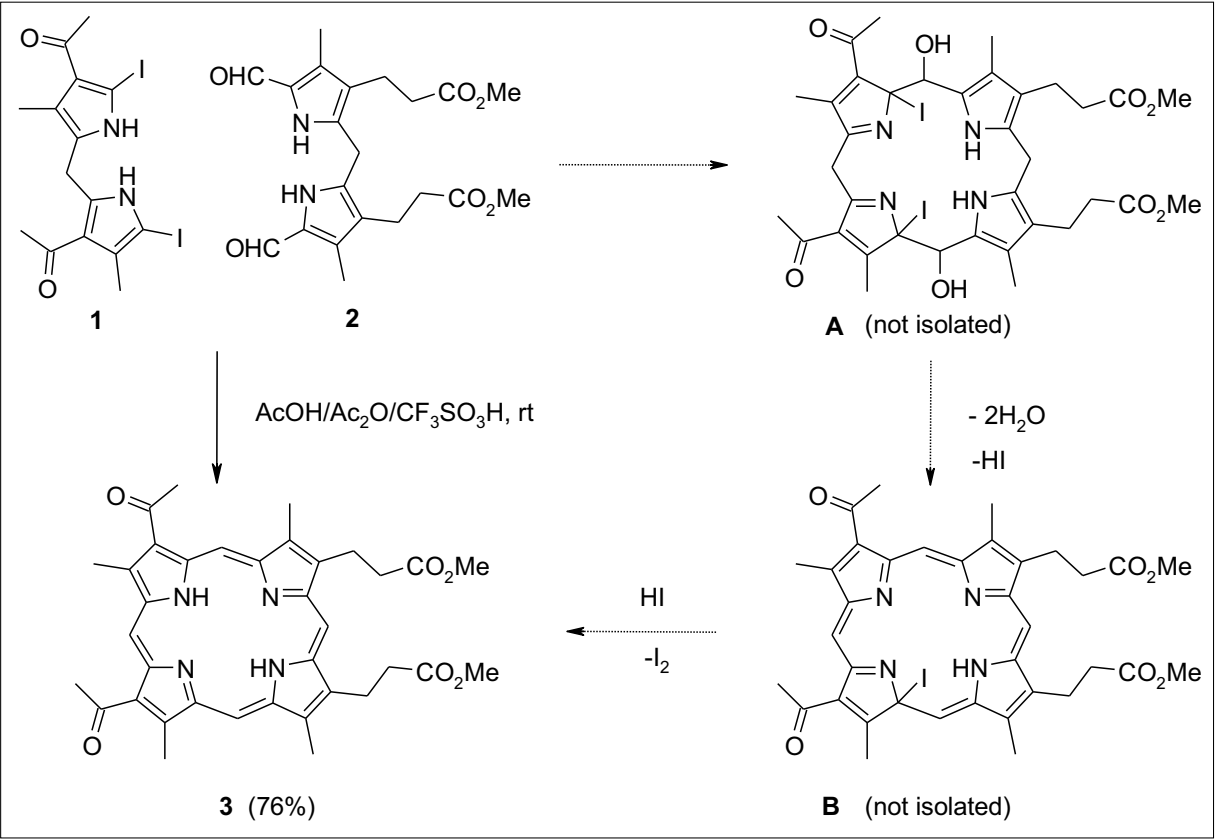

Scheme 1.

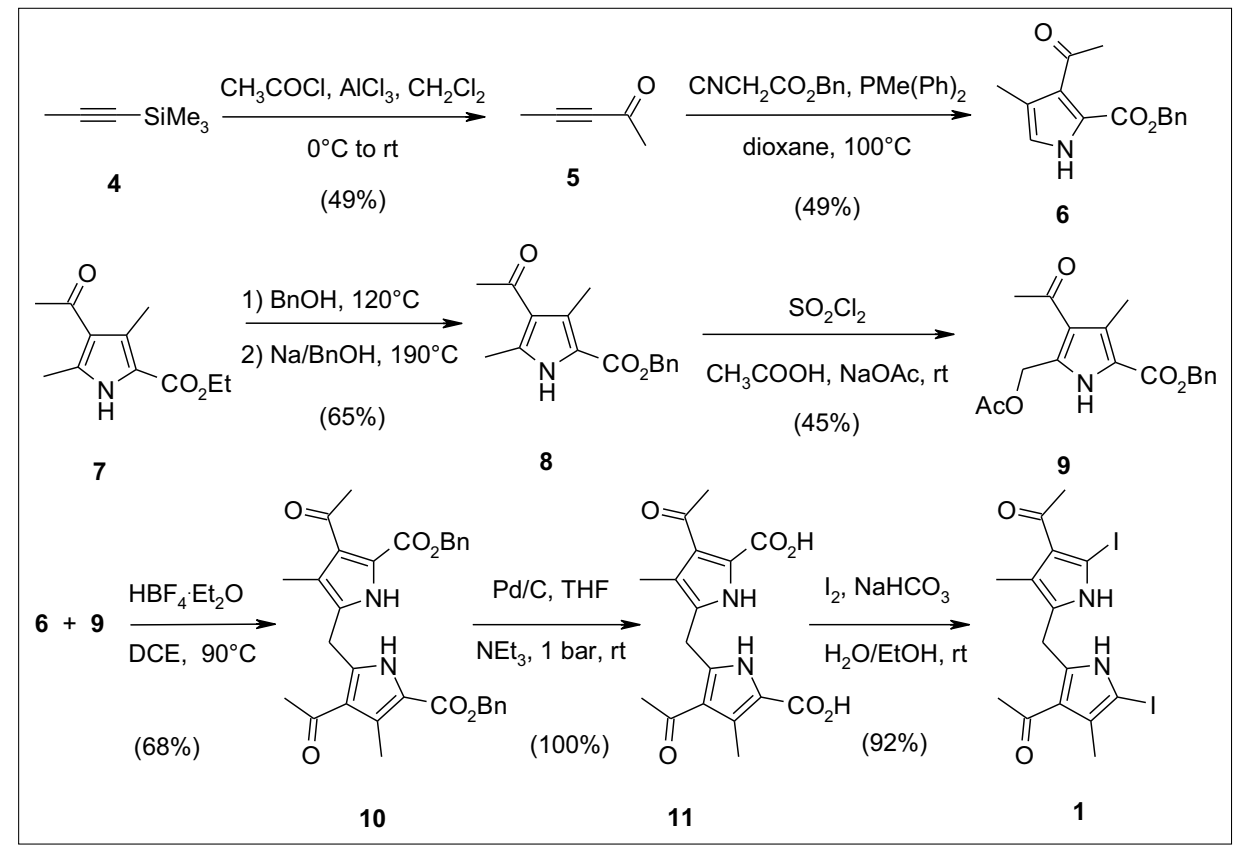

Scheme 2 .

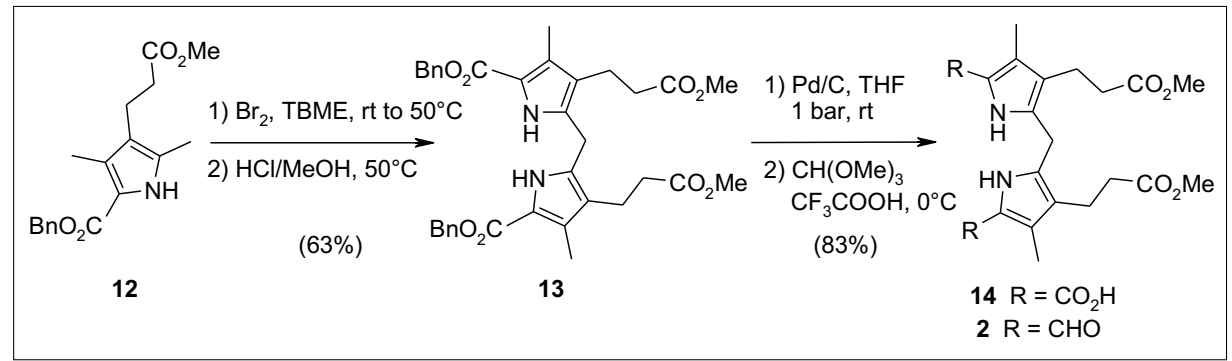

Scheme 3.

\section{Conclusions}

Protoporphyrin IX dimethyl ester 16 and its disodium salt $\mathbf{1 7}$ can be prepared from commercially available starting mate- rials via a modified MacDonald procedure. The key step is the condensation reaction of the diiodo dipyrrylmethane $\mathbf{1}$ with the dialdehyde $\mathbf{2}$ which directly furnishes the porphyrin 3 without the need of oxidation 


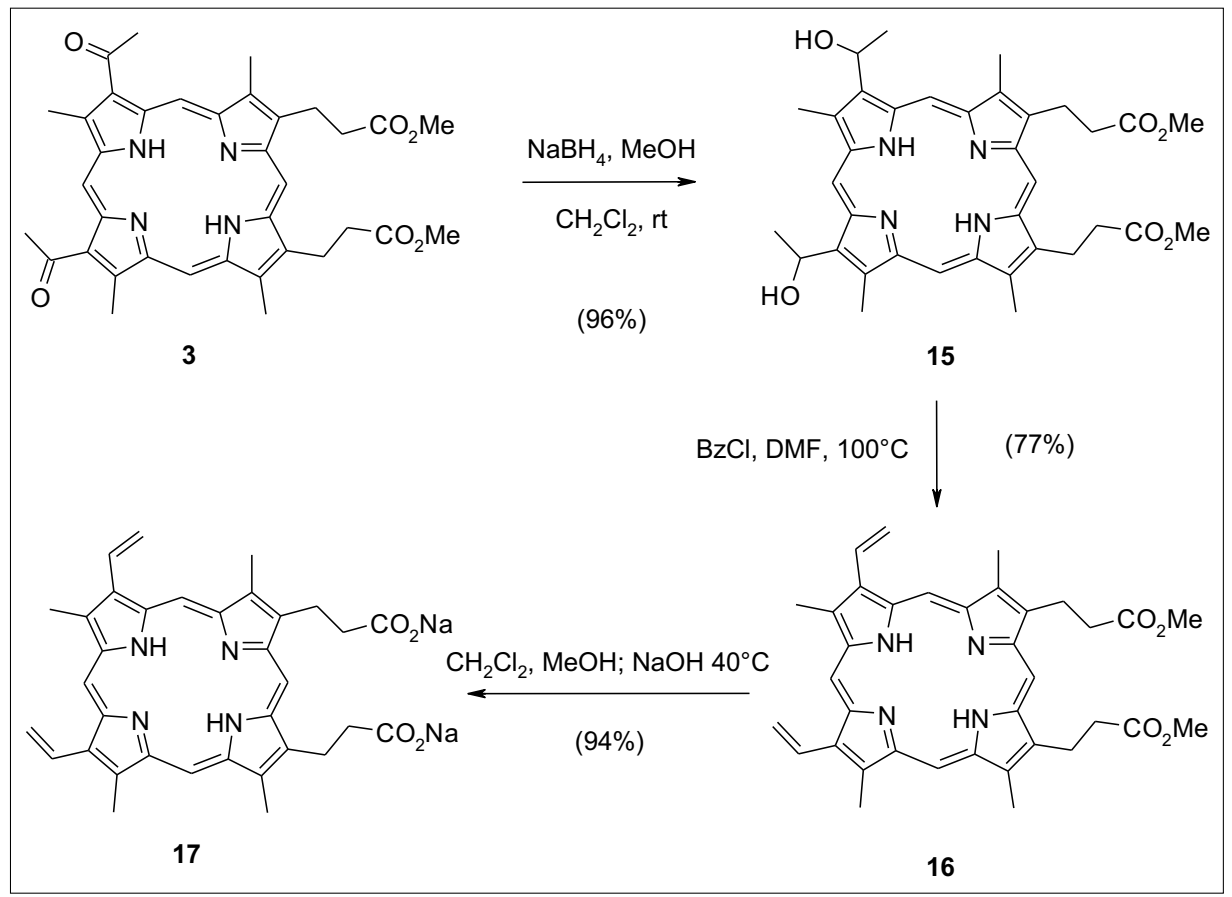

Scheme 4.

of a dihydroporphyrin intermediate. Both dipyrrylmethane units can be prepared on multi-100 $\mathrm{g}$ scale in excellent purity and satisfactory yield without the need of chromatography.

\section{Acknowledgements}

We thank Sanofi-Pasteur for the fruitful collaboration and the permission to publish our results.

Received: October 8, 2012

[1] The original procedure was first reported by M. Schalfejew, Hoppe Seylers Zeitschrift 1900, 30, 390 (see also Ullmann, Encyklopädie der technischen Chemie, IV edition, 1976, Vol. 11, p. 129). An improved procedure is described by H. Schulze, US-Pat. 4761472 (1988, assigned to BASF). About 3-4 g of heme are obtained from 11 of oxblood.
[2] A. H. Jackson, K. R. Rao, M. Wilkins, J. Chem. Soc., Perkin Trans. 1 1987, 307; W. Chen, H. Yang, D. Xu Zhnongguo Yiyao Gongye Zazhi 2000, 31, 293. CAN 134: 193262.

[3] Official J. Europ. Union, C24, 28.1.2004, p. 6.

[4] R. Chong, P. S. Clezy, A. J. Liepa, A. W. Nichol, Austr. J. Chem. 1969, 22, 229; A. H. Jackson, R. K. Pandey, J. Chem. Soc., Perkin Trans. 1 1987, 299.

[5] G. P. Arenault, E. Bullock, S. F. MacDonald, J. Am. Chem. Soc. 1960, 82, 4384; M. Chakrabarty, J. Ind. Chem. Soc. 2001, 78, 761; P. S. Clezy, V. Diakiw, Austr. J. Chem. 1975, 28, 2703.

[6] C.-H. Lee, F. Li, K. Iwamoto, J. Dadok, A. A. Bothner-By, J. S. Lindsay, Tetrahedron 1995, 51,11645 .

[7] Indeed, $I_{2}$ could be observed when the reaction solution was heated in the rotovap. Furthermore, small amounts of a compound could be isolated via chromatography which showed the appropriate mass corresponding to intermediate $\mathbf{B}$ and reacted to give $\mathbf{3}$ in presence of HI.

[8] For experimental details see P. Martin, M. Müller, D. Flubacher, A. Boudier, H. U. Blaser, D. Spielvogel, Org. Process Res. Devel. 2010 , 14, 799. 